MATEC Web of Conferences 42, 02002 (2016)

DOI: $10.1051 /$ matecconf/20164202002

(C) Owned by the authors, published by EDP Sciences, 2016

\title{
Mechanisms of Minimum Skew Angle Actuation for Hard Disk Drives
}

\author{
Zhimin $\mathrm{He}^{\mathrm{a}}$, Jianqiang Mou, and Kheong Sann Chan \\ $A^{*}$ Star, Data Storage Institute, 2 Fusionopolis Way, \#08-01, Innovis, Singapore 138634
}

\begin{abstract}
The utilization of voice coil motor (VCM) rotary actuator in hard disk drives (HDDs) results in skew angles between the read/write head and disk rotation direction. The system performance and achievable capacity of hard disk drives are excessively sensitive to the skew angle [1-3]. The difference in skew angle, between the ID to the OD can be as large as more than 30 degrees in conventional 3.5" and 2.5" VCM actuated HDDs. This paper presents three mechanisms, namely, a slant arm/suspension mechanism, a guider track mechanism, and a 4 link mechanism which can be designed to achieve minimum skew angle actuations for hard disk drives. The slant arm/suspension mechanism reduces the skew angle to \pm 2 degrees in rotary actuated systems. The guider track mechanism further achieves the skew angle as zero degree theoretically. As an approximation of the guider track mechanism, the 4 link actuation mechanism reduces the skew angle to 0.4 degree. Their system dynamic behaviors are studied numerically using finite element modelling. Some of designs are prototyped and experimentally investigated.
\end{abstract}

\section{Introduction}

In HDDs, circular data tracks are accessed by read/write elements mounted on a supporting arm/suspension, which is swung by a voice coil motor (VCM). The utilization of VCM rotary actuation results in a skewed actuation between the read/write element and the track that the head is following. The skew angle variations can be $25 \sim 35^{\circ}$ from the disk ID to OD for typical $2.5^{\prime \prime}$ and $3.5^{\prime \prime}$ HDDs [1]. A large skewed actuation affects the slider's flying performance and off-track capability, limiting the performance of HDDs. It causes an increase in side reading and writing and complicates the position error signal (PES) calibration process in the HDD servo loop. The soft magnetic under-layer in perpendicular recording, while improving the perpendicular write efficiency, exacerbates the problems with head skew and stray fields [2]. Therefore, the adverse effects due to head skew are more significant for perpendicular recording. Zhou [3] revealed the effects of the head pole aspect ratio and skew angle on areal density in perpendicular recording. The track density and areal density can be increased more than $20 \%$ when the skew is $0^{\circ}$ compared to that of $15^{\circ}$ skew.

Previously IBM proposed a parallel flexural in-line actuator for magnetic recording disk drives which has a small skew angle of $1.5^{\circ}$ [4]. However, this design makes a significant change in the actuator structure compared to current actuator designs. A slant arm/suspension actuator can be designed and optimized to achieve skew angle as small as $\pm 2^{\circ}$ in rotary actuated systems [1]. The length of the arm/suspension assembly has been increased about $20 \%$, which leads to $20-30 \%$ drop of resonance frequencies of the system compared to a conventional design. A parallel link mechanism has been proposed by Seagate Technology to reduce the skew angle variation [5], but the link position and dimensions have yet to be optimized. More recently, a MEMS based rotary actuator was presented for skew angle compensation of HDDs [6]. The MEMS based actuator can rotate the read/write head around $\pm 13^{\circ}$. It needs a high driving force of 75 volts, and is with slow response of $1.67 \% \mathrm{~ms}$.

In this paper, we summarize 3 different mechanisms which attain minimum skew actuation for hard disk drives. Firstly a slant arm/suspension mechanism, as described in [1], with a conventional VCM actuation mechanism, has a small skew actuation with the skew angle as $\pm 2^{\circ}$. In the second, a guider track mechanism is presented to theoretically have a skew angle as zero. The third, a 4-links actuation mechanism, which is an approximation of the guider track mechanism, can be designed to achieve skew angle as small as 0.4 degree. The dynamic performances of the mechanisms are evaluated with finite element modelling and experimental investigation. The benefits of small skew actuation on areal density for the presented mechanisms and their dynamic performances are compared with a conventional actuation mechanism and their issues are discussed.

\section{Mechanisms of small skew angle actuation for hard disk drives}

\subsection{Rotary actuator with slant arm/suspension structure}

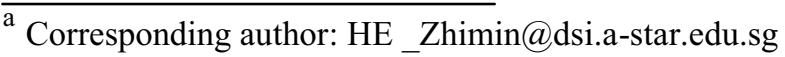


Here we apply the configuration of slant arm/suspension [2] to a 2.5" form factor hard disk drive. Figure 1 shows a layout of a typical 2.5" form factor HDD. The distance from the pivot centre to the spindle centre is as $D=39.13$ $\mathrm{mm}$; the radius in the inner diameter, $r_{\text {id }}=12.5 \mathrm{~mm}$; the radius in the outer diameter, $r_{\text {od }}=32.5 \mathrm{~mm}$. $L_{0}$ stands for the distance between the pivot centre to the read/write element. It is to be optimized. Figure 2 shows the optimization of arm length ratio to have minimum skew angle range from ID to OD. When the arm length ratio is 1.13 , the skew angle range is minimized as $4^{\circ}$.

To enable minimum skew angle actuation, we can further slant the arm or suspension, as shown in Figure 3. In this case, the suspension length $L_{\mathrm{s}}$ is fixed, the slant angle $\beta$, will depend on the actual arm length, $L_{a}$. We optimize the arm length, $L_{a}$, such that the absolute skew angles from the inner diameter to outer diameter, are within $\pm 2^{\circ}$. When the suspension is slanted, the optimized slant angle $\beta$ is calculated as $42^{\circ}$.

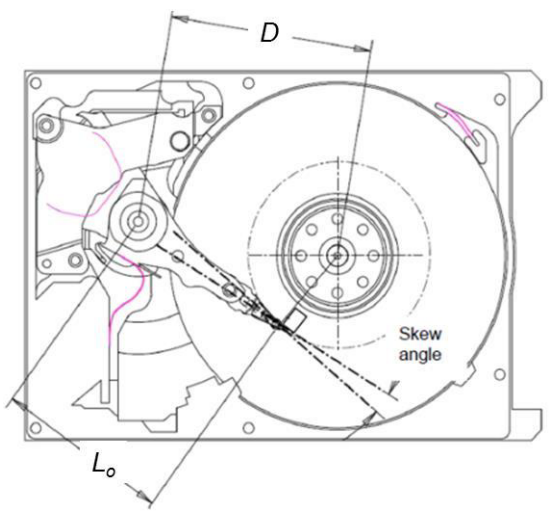

Figure 1. A typical 2.5" HDD layout for skew angle calculation and optimization.

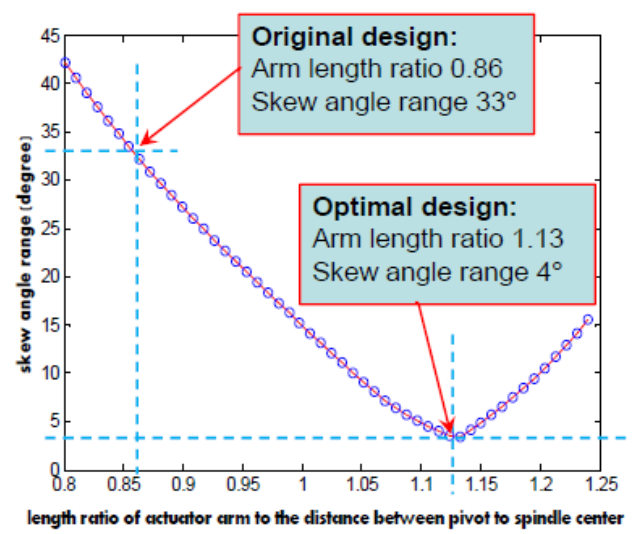

Figure 2. Optimization of arm length ratio for minimum skew angle range.

\section{$2.2 \mathrm{VCM}$ actuator with a guider track}

To ensure absolute zero skew actuation a guider track skew correcting system that includes a rotational suspension integrated with the guider link and guider track. The guiding track profile can be determined based on the disk drive geometry and the guider link length, such that the head skew angle is always zero as the magnetic head element is moved from ID to OD.

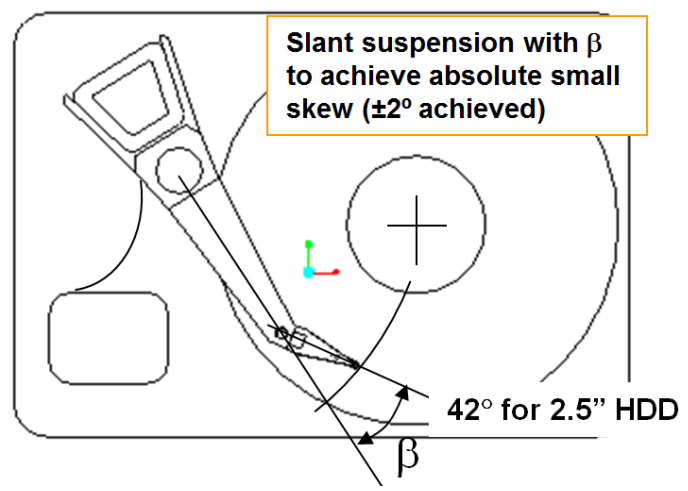

Figure 3. A layout of HDD with slant arm/suspension structure.

Figure 4 shows a schematic of a guider track actuation mechanism for a disk drive system to achieve zero skew actuation. In Figure 4, o and $\mathrm{o}_{1}$ are the center of disk rotation, and the VCM pivot center respectively. $l_{a}$ and $l_{s}$ are the arm length and the suspension length respectively. $r$ is the radius of the circle where the magnetic head element is located at with respect to the spindle centre. The suspension is integrated with a guider link AC as a whole part. The angle between the suspension direction $\mathrm{AB}$ and the guider link $\mathrm{AC}$ is fixed. Here without loss of generality, we let $\angle \mathrm{BAC}=90^{\circ}$ (though it can be an angle other than $90^{\circ}$ ). If we constrain the head skew angle to be zero, then $\angle \mathrm{oBA}$ should be $90^{\circ}$. Based on the geometric relationship, we establish the following relations,

$$
\begin{aligned}
& d=l_{a} \sin \theta_{1}+l_{s} \cos \theta+r \sin \theta \\
& r^{2}+l_{s}^{2}=d^{2}+l_{a}^{2}-2 d l_{a} \sin \theta_{1} \\
& x=l_{a} \cos \theta_{1}+l \cos \theta \\
& y=l_{a} \sin \theta_{1}-l \sin \theta
\end{aligned}
$$

From (3)

$$
\sin \theta_{1}=\frac{d^{2}+l_{a}{ }^{2}-r^{2}-l_{s}{ }^{2}}{2 d l_{a}}
$$

For every location of the head element, i.e., different $r, \theta_{1}$ is determined by (5), and $\theta$ is determined by (1). The guider track profile $(x, y)$ can be determined by (3) and (4).

An implementation of the guider track actuation mechanism in a hard disk drive is shown in Figure 5. The guider track is mounted on the disk drive base. The guider link and the head suspension assembly (HSA) form an integrated part that can be pivotally rotate at the end of the arm. A shaft fixedly connects the multiple guider links, which correspond to multiple head suspension assemblies (HSAs). A rolling element including a pivot assembled at one end of the shaft rolls along the guider track such that the head skew angle is always zero while the magnetic head element moves from the disk outer diameter to the inner diameter.

An approximation of the guider track mechanism is a 4link actuation mechanism [1]. The 4-links actuation mechanism includes a conventional VCM actuator, an 
arm, a rotational suspension with a coupler link, and a follower link. The fixed location of the follower link, the lengths of the links can be designed and optimized such that the skew angle between the suspension longitudinal direction and disk rotating direction as small as possible. The 4 link mechanism can be analysed and optimized such that the skew angle as small as 0.4 degree for a 3.5" HDD, which has been described in [1].

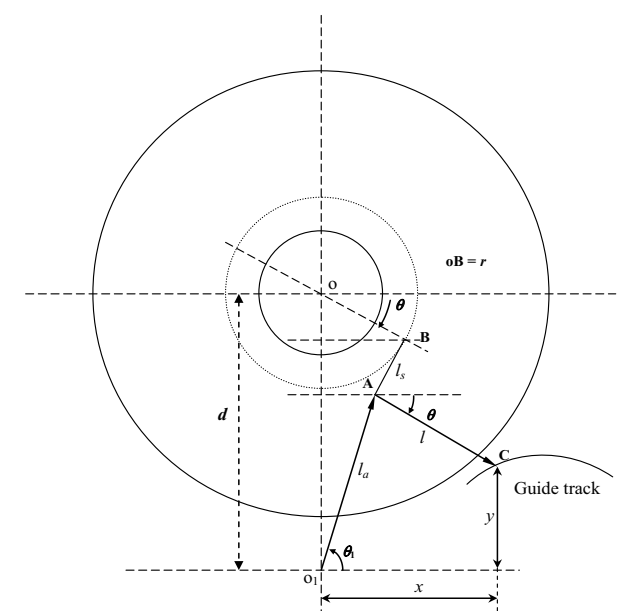

Figure 4. Schematic of a disk drive actuator system with a guider track.

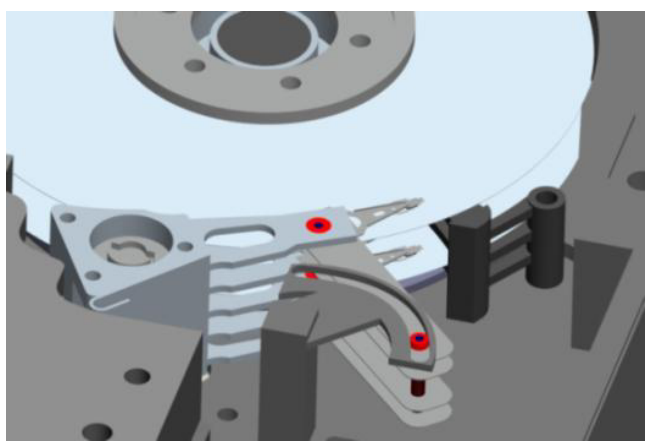

Figure 5. Hard disk drive actuator system with a guider track actuation mechanism for zero skew actuation (OD).

\section{Dynamic performance simulation}

\subsection{Slant arm suspension mechanism}

Finite Element (FE) modelling with ANSYS is employed to study the dynamic performance of the mechanisms for small skew angle actuation. Figure 6 shows a full structural FE model of a conventional 2.5" HDD. The model includes the disk, spindle motor and fluid dynamic bearing (FDB), VCM actuator, pivot, Head Gimbal Assembly (HGA), base plate and top cover. The HGA including the slider with head, load beam and flexure, is integrated with the actuator through a mounting plate and assembled with the disk through an air bearing. The full structural FE model of the HDD has been validated in by comparing frequency response simulation results with experimental results using a Polytec 3D laser Doppler Vibrometer (LDV) [8].

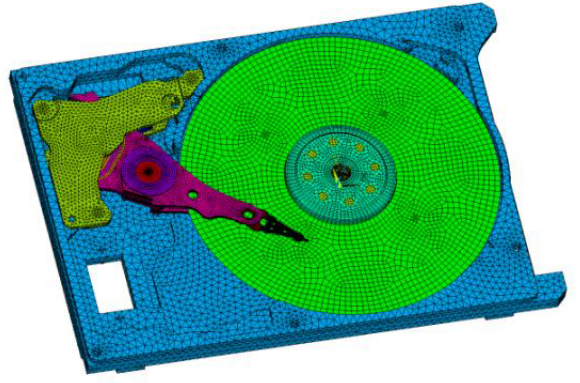

Figure 6. Structural FE model of a conventional 2.5" HDD.

Figure 7 shows the FE model of the slant arm/suspension structure design. The simulation results of head off-track displacement frequency response of the slant arm/suspension design and the conventional design are shown in Figure 8. The first off-track dominant vibration mode of the slant arm/suspension design is shifted to a lower frequency at $7760 \mathrm{~Hz}$, and an extra mode is introduced at the $7150 \mathrm{~Hz}$. The analysis results demonstrate that both the elongated arm/suspension design and slant arm/suspension design for small skew angle actuation will make the resonant frequency of the off-track dominant vibration mode nearly $20 \%$ smaller, which may deteriorate the servo bandwidth of the actuator.

\subsection{Guider track mechanism}

The FE model of the guider track design is shown in Figure 9. The simulation results of head off-track displacement frequency response of the guider track design and the conventional design are shown in Figure 10. It is found that the first off-track dominant mode at frequency $9600 \mathrm{~Hz}$ of the original design is split into two modes at frequency $8700 \mathrm{~Hz}$ and $10300 \mathrm{~Hz}$ respectively. On the other hand, the vibration modes at $13000 \mathrm{~Hz}$ and $20000 \mathrm{~Hz}$ of the original design are compressed in amplitudes after introduction of the guider track.

The modal analysis results show that the first mode is the actuation mode at frequency $0.9 \mathrm{~Hz}$, which demonstrates the actuator with the guider track design is workable. The first off-track dominant vibration mode of the HDD is reduced to $8700 \mathrm{~Hz}$, which is due to the extra mass introduced by the track link and no resistance along the track The second off-track dominant vibration mode of the HDD is increased to $10300 \mathrm{~Hz}$, which is due to the track link resisted in the direction perpendicular to the track. The resonant frequency of the first off-track mode can be improved by increase the stiffness of pivot bearing and optimization of the original arm design.

The results of FE modeling for a 4-link actuation mechanism have been revealed in [1]. Similar to the guider track mechanism, the first in-plane resonance mode of the actuator is shifted lower compared to conventional actuator. The second in-plane dominant mode is increased due to the system stiffness increase in the down track direction. Vibration modes in high frequency range are suppressed in amplitudes after introduction of the four links. In summary, the resonant frequency of the first off-track dominant vibration mode 
of the HDD will be reduced after introduction of the mechanisms for small skew angle actuation. Among the three actuation mechanisms, the guider track design has the minimal impact on the degradation at resonance performance along the tracking direction compared to a conventional actuation mechanism.

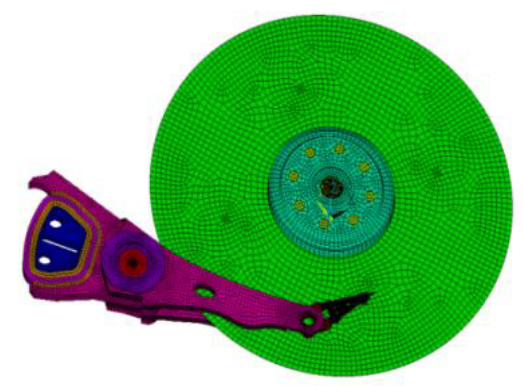

Figure 7. Slant arm/suspension structure design.
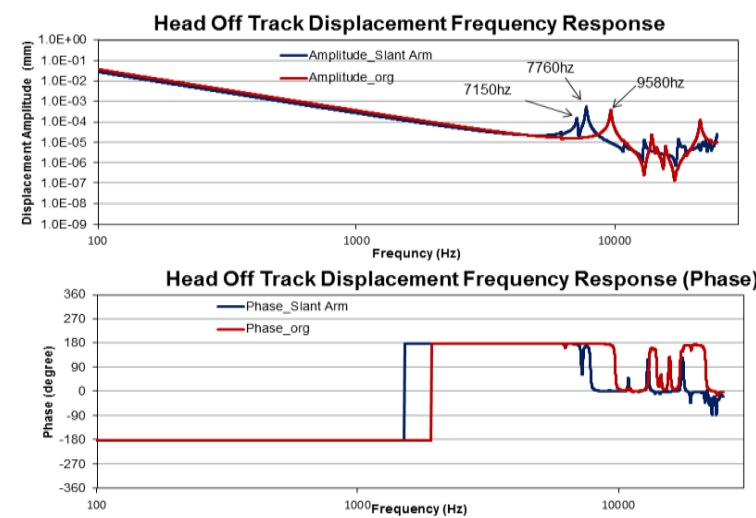

Figure 8. Frequency response of the slant arm/suspension design and conventional design.

\section{Experiment and discussion}

\subsection{Experiment}

Prototypes made of a typical 3.5" disk drive actuator arm and suspension for the actuator assemblies with and without slant arm/suspension have been presented in [2]. The measured frequency response of the prototypes does not show significant differences for the actuator assemblies with and without slant arm/suspension. However, the length ratio for a minimal skew range has been increased to 1.11 from about 0.93 in the current actuator assembly for a 3.5" HDD. This causes the resonance frequency for the arm sway mode to drop $20 \sim 30 \%$ from the conventional design. Therefore, a small skew actuator design, which does not sacrifice bandwidth, is required.

Another experimental investigation is the comparison of the response speed between a 4 link actuation mechanism with that of a conventional actuation mechanism [1]. The voice coil motor (VCM) is injected with driving voltage \pm 1 volt from a dynamic signal analyzer (DSA). Both actuation mechanisms move their suspension tips from
OD to ID. Meanwhile, a high speed camera is used to capture the movement of the suspension at a very slow speed of $1000 \mathrm{~Hz}$, or 1 millisecond/frame.

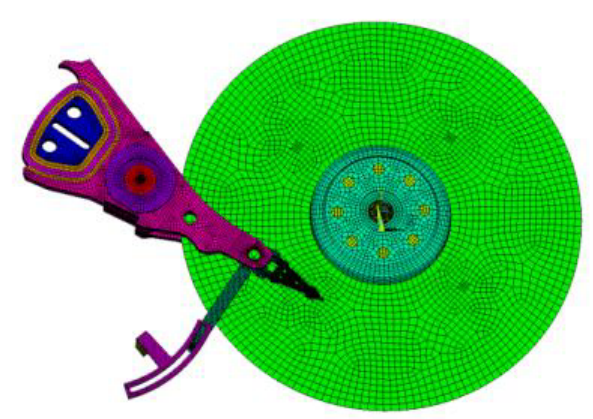

Figure 9. The guider track actuation design.
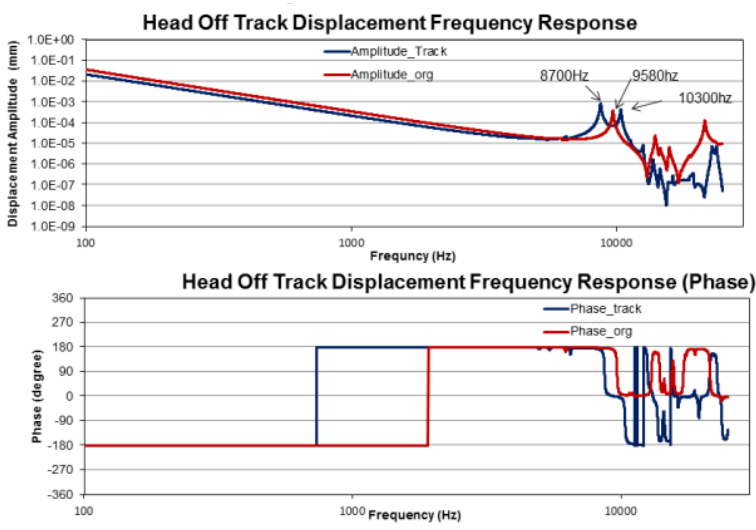

Figure 10. Frequency response of the guider track design and conventional design.

It is found that for the conventional actuator assembly, the suspension tip linear velocity is 1.4 times of that at the arm tip. However, for the 4 link actuation mechanism, the linear velocity at the suspension tip is 0.9 times of the velocity at its arm tip. Therefore, the speed of the suspension for the 4 link actuation mechanism is about $(1.4-0.9) \times 100 \%=50 \%$ slower than the original actuation mechanism.

\subsection{Comparison of skew angle effects on areal density}

Based on the analysis of head pole aspect ratio and skew angle effects on areal density in perpendicular recording [4], the benefits of small skew gain on areal density for a slant suspension assembly and the guider track actuator assembly are compared and the issues are discussed as shown in Table 1. For the slant suspension assembly, the actuator arm length is to be increased by about $30 \%$ of the original design, resulting in the in-plane first resonance frequency dropping by 20 to $30 \%$, leading to degraded control bandwidth and shock resistance. The remedies include reducing the actuator inertia, specifying the proper suspension to arm length ratio, and designing high bandwidth VCM arm and suspension structures [1], and so on. In addition, introducing a secondary stage actuator for dual stage control of the head element is an 
effective way to increase the actuator servo bandwidth $[11,12]$. Furthermore, reducing vibration due to air flow, spindle motor, disk, etc., can relax the requirements on servo bandwidth [13.]

Table 1. Comparison and summary of the effects of different skew angles.

\begin{tabular}{l|c|c|l}
\hline \multicolumn{1}{c|}{ Design } & $\begin{array}{c}\text { Skew } \\
\text { angle } \\
\text { range }\end{array}$ & $\begin{array}{c}\text { Potential } \\
\text { areal density } \\
\text { gain }\end{array}$ & \multicolumn{1}{c}{ Remarks } \\
\hline $\begin{array}{l}\text { Conventional } \\
\text { actuator }\end{array}$ & $25 \sim 35^{\circ}$ & ----- & $\begin{array}{l}\text { The adverse effects of } \\
\text { skew angle on areal } \\
\text { density and track } \\
\text { density }\end{array}$ \\
\hline $\begin{array}{l}\text { Slant } \\
\text { suspension }\end{array}$ & $4^{\circ}\left( \pm 2^{\circ}\right)$ & $10 \sim 16 \%$ & $\begin{array}{l}\text { Increase of actuator arm } \\
\text { length by 30\% resulting } \\
\text { in arm in-plane and out- } \\
\text { of-plane resonance } \\
\text { frequencies drop. }\end{array}$ \\
\hline $\begin{array}{l}\text { Guider track } \\
\text { mechanism }\end{array}$ & $0^{\circ}$ & $12 \sim 20 \%$ & $\begin{array}{l}\text { Issues of frictions due to } \\
\text { additional joints and } \\
\text { challenges of system } \\
\text { integration in HDDs. }\end{array}$ \\
\hline
\end{tabular}

For the guider actuation mechanism, there are dynamic issues, such as frictions in the additional joints may cause the system control difficult. Design of low friction and high stiffness joints or application of micro actuators can overcome the problems. In another aspect, implementing the mechanism in a real hard disk drive will change the structure of the existing actuation system, bringing in challenges in system integration.

\section{Conclusions}

A disk drive actuator can be designed with a skew angle of \pm 2 degrees by extending the actuator arm length to a certain point and slanting the suspension or head assembly. Elongating the arm and slanting suspension make the resonant frequency of the off-track dominant vibration mode drop nearly $20 \%$. There is no significant change in the dynamic performance for the actuator from slanting the suspension.

A guider track mechanism for the actuation assembly achieves the skew angle as zero degree. Its dynamic offtrack frequency drops around $10 \%$ compared a to conventional actuator assembly. An approximation of the guider track mechanism is a 4 four link actuation mechanism which can be designed and optimized to achieve 0.4 degree skew angle variation. With proper design of the joints and links, the resonance performance along the tracking direction is not degraded compared to a conventional actuation mechanism. With the proposed small skew actuation mechanisms, the potential areal density gain is projected at $12 \sim 20 \%$ compared to a conventional actuation mechanism. However, there exist system integration challenges for implementing the guider track and 4 link actuation mechanisms in HDDs.

\section{ACKNOWLEDGMENT}

The authors wish to thank colleagues Mr. Lam Suet Hoi for providing support in Matlab debugging, and See Bong Long in performing CAD modeling.

\section{REFERENCES}

1. Z.M. He, J.Q. Mou, K. S. Chan, S.H. Lam and W. Lin, "A near zero skew actuation mechanism for hard disk drives", Microsyst Technol 21:131-137 (2015)

2. Z.M. He, E.H. Ong, and G.X. Guo, "Optimization of a magnetic disk drive actuator with small skew actuation", J. of Appl. Phys., AIP, 91(1), 8709-8711 (2002)

3. B.M. Lairson, S.E. Lambert, H. Nguy, L. Nguyen, A. Wallash, T. Huang, J. Adler, "Disk drive integration issues for perpendicular recording", IEEE Trans. Magn., 37(4), 1223-1227(2001)

4. H. Zhou, "Effects of head pole aspect ratio and skew angle on areal density in perpendicular recording", IEEE Trans. Magn., 40(4), 2395-2397 (2004)

5. T. J. Chainer, W.J. Sohn, M. Sri-Jayantha, D.H. Brown, N.C. Apuzzo, "A Flexural In-Line Actuator for Magnetic Recording Disk Drives”, IEEE Trans. Magn., 27(6), 52955297(1991)

6. Seagate Technology LLC, Hard Drives Actuator with Reduced Skew Variation, US patent: 7,072,147 B2.

7. E. Sarajlic, C. Yamahata, M. Cordero, H. Fujita, "Threephase electrostatic rotary stepper motor for skew angle compensation", Asia Pacific Magnetic Recording Conference (APMRC), Singapore, Digest, AB-1, November 10-12 (2010)

8. J.Q. Mou, Q.D. Zhang, K. Sundaravadivelu, N.Y. Liu, and E. H. Ong, "Reduction of flow induced vibration in hard disk drive", Proceedings of ASME IMECE2010, IMECE2010-37649, Vancouver CANADA, November $12-18(2010)$

9. E. M. Jayson, J. Murphy, P.W. Smith, and F.E. Talke, "Effects of air bearing stiffness on a hard disk drive subject to shock and vibration," ASME J. Tribol., 125(2), 343-349 (2003)

10. Q. H. Zeng, and D. B. Bogy, "Numerical simulation of shock response of disk-suspension-slider air bearing systems in hard disk drives", Microsyst. Technol. 18: 289$296(2002)$

11. Z.M. He, H.T. Loh, and E.H. Ong, "Reliability Evaluation of Piezoelectric Micro-Actuators With Application in Hard Disk Drives”, IEEE Trans. On Magn., 44(11), 3722-3724 (2008)

12. G.X. Guo, Q. Hao, and T.S. Low, "A dual-stage control design for high track per inch hard disk drives," IEEE Trans. Magn., 37 (2), 860-865 (2001)

13. Z.M. HE, C.L. Du, J.Q. Mou, and E.H. Ong, "Reducing position error signal (PES) due to disk vibration using an air shroud", Journal of Sound and Vibration (311), 874 885 (2008) 